\title{
Programa individualizado de exercícios para incontinência urinária executado no espaço domiciliar
}

\author{
Individualized exercise program for urinary incontinence performed in the home space
}

\author{
Evelise Moraes Berlezi ${ }^{1}$, Marília Martins ${ }^{2}$, Daniela Zeni Dreher ${ }^{3}$
}

${ }^{1}$ Fisioterapeuta. Doutora em Gerontologia Biomédica. Docente do Programa de Pós-Graduação em Atenção Integral à Saúde e do Departamento de Ciências da Vida da Universidade Regional do Noroeste do Estado do Rio Grande do Sul (UNIJUI). Coordenadora do projeto "Estudo Multidimensional de Mulheres Pós-menopausa do Município de Catuípe/RS”. Ijuí, RS.

2 Graduanda do curso de fisioterapia da UNIJUÍ. Bolsista do Programa Institucional de Bolsas de Iniciação Científica do CNPq. Ijuí, RS.

${ }^{3}$ Fisioterapeuta. Mestre em Engenharia de Produção. Docente do Departamento de Ciências da Vida da UNIJUI. Ijuí, RS.

Trabalho vinculado à pesquisa institucional "Estudo multidimensional de mulheres pós-menopausa do Município de Catuípe/RS" da Universidade Regional do Noroeste do Estado do Rio Grande do Sul.

\section{RESUMO}

Objetivos: Avaliar a eficiência de um programa individualizado de exercícios para incontinência urinária executado no espaço domiciliar sob supervisão e avaliação sistemática do fisioterapeuta.

Métodos: A população do estudo foi constituída por mulheres pós-menopausa, entre 50 a 65 anos de idade, residentes na área urbana do município de Catuípe, Rio Grande do Sul. Para a seleção da amostra consideraram-se como critérios de inclusão o diagnóstico urofuncional de incontinência urinária e a adesão ao protocolo de intervenção, constituído pelo treinamento dos músculos do assoalho pélvico e ginástica hipopressiva executado durante 12 semanas, com supervisão e acompanhamento sistemático pelo fisioterapeuta. A avaliação foi realizada pré e pós-intervenção e constituiu-se em anamnese e avaliação funcional da musculatura do assoalho pélvico.

Resultados: A amostra foi constituída por cinco mulheres. O protocolo aplicado promoveu o incremento da força muscular, tempo de sustentação da contração muscular, aumento da velocidade de contração e número de repetições da musculatura do assoalho pélvico. Os dados mostram a redução de $80 \%$ de queixas de perda de urina ao esforço; redução de $40 \%$ na sensação de vontade de urinar em situações de estresse, frio, barulho de torneira e mãos na água; e na continência de $100 \%$ das mulheres em situação de instabilidade.

Conclusões: Os resultados do estudo evidenciam que o programa de exercícios individualizado executado no espaço domiciliar, sob supervisão e avaliação sistemática do fisioterapeuta, foi eficiente no tratamento da incontinência urinária.

DESCRITORES: INCONTINÊNCIA URINÁRIA; MULHERES; ATENÇÃO PRIMÁRIA.

\begin{abstract}
Aims: To evaluate the effectiveness of an individualized exercise program for urinary incontinence performed in the home space under supervision and systematic evaluation of the physiotherapist.

Methods: The study population consisted of postmenopausal women aged 50 to 65 years, living in the urban area of the municipality of Catuípe, Rio Grande do Sul. The criteria of inclusion considered for selection of the sample were urofuncional urinary incontinence and adherence to the intervention protocol, consisting of training of the pelvic floor muscles and hipopressiva gym run for 12 weeks, with systematic monitoring and supervision by the physiotherapist. The evaluation was performed pre-and post-intervention and was constituted by history and functional evaluation of the pelvic floor muscles.

Results: The sample consisted of five women. The protocol used has favored the increase of muscle strength, time of sustained muscle contraction, increased contraction speed and number of repetitions of the pelvic floor muscles. The data show an $80 \%$ reduction in complaints of loss of urine on exertion, $40 \%$ reduction in the sensation of the urge to urinate in situations of stress, cold, noise tap and hands in water, and $100 \%$ continence in women in instability.

Conclusions: The study results show that individualized home exercise program performed in home space and under the supervision of a physiotherapist systematic review was effective in the treatment of urinary incontinence.
\end{abstract}

KEY WORDS: URINARY INCONTINENCE; WOMEN; PRIMARY HEALTH CARE. 


\section{INTRODUÇÃO}

A incontinência urinária (IU) é definida como a perda involuntária de urina em locais e situações inadequadas. Essa condição traz implicações sociais, causa desconforto e perda da autoconfiança, prejudica a vida sexual e interfere negativamente na qualidade de vida de muitas mulheres, além de representar um problema de saúde pública. Segundo estimativas da Sociedade Brasileira de Urologia, uma em cada 25 pessoas desenvolve incontinência urinária e $40 \%$ das mulheres começam a perder urina de forma involuntária após a menopausa. ${ }^{1-4}$

Muitas mulheres acreditam que a perda de urina ao realizar um esforço é natural e inerente ao envelhecimento. Uma das causas nessa etapa da vida pode ser o hipoestrogenismo, por interferir no tônus da musculatura do assoalho pélvico. Contudo, independentemente da causa da IU, há tratamentos disponíveis com efetividade comprovada, sejam eles cirúrgicos ou conservadores. ${ }^{1,3}$

Na modalidade conservadora, o treinamento dos músculos do assoalho pélvico (TMAP) e a ginástica hipopressiva $(\mathrm{GH})$ são técnicas da fisioterapia que auxiliam no tratamento da IU. O TMAP é o tratamento de primeira opção por ser um método seguro e eficaz, que promove a melhora do tônus muscular e confere suporte às estruturas perineais por meio da contração de seus músculos de forma voluntária. A contração é percebida quando a atividade muscular causa um movimento de compressão e elevação do assoalho pélvico. ${ }^{5,6}$

Por sua vez, a GH é uma técnica que se baseia na contração ativa da musculatura abdominal provocando a elevação dos órgãos pélvicos na direção do diafragma respiratório, com contração supostamente reflexa dos músculos do assoalho pélvico (MAP). ${ }^{7}$ Essa contração involuntária pode ser uma forma de treinamento dos MAP, pois estes podem ser treinados indiretamente pela contração do músculo transverso abdominal. ${ }^{8} \mathrm{~A}$ GH complementa os exercícios para os MAP e auxilia pacientes que não têm consciência ou apresentem dificuldade em realizar a contração perineal. ${ }^{7}$

As técnicas supracitadas são utilizadas em clínicas de fisioterapia, contudo testá-las no ambiente domiciliar é de suma importância para verificar a sua eficácia, em função da alta demanda em serviços públicos de saúde. A necessidade de ampliar a atenção à saúde da mulher por meio de estratégias que possam ser reproduzidas pelas equipes de saúde justifica a proposição de um protocolo de exercícios domiciliares que associa os dois métodos conservadores, com acompanhamento sistemático realizado por um fisioterapeuta e prescrição personalizada de acordo com o esquema PERFECT $^{9}$ (power, endurance, repetition, fast, every, contractions, timed), que tem por finalidade avaliar a musculatura do assoalho pélvico quanto à força, duração, repetições e número de repetições rápidas das contrações.

Destaca-se que a atenção domiciliar representa uma estratégia de atenção à saúde que engloba mais do que o fornecimento de um tratamento padrão. É um método aplicado ao cliente com o objetivo de enfatizar sua autonomia e realçar suas habilidades em seu próprio ambiente $-\mathrm{o}$ domicílio. ${ }^{10} \mathrm{~A}$ atenção domiciliar à saúde é considerada um dos meios de se obter do indivíduo, da família e da comunidade a participação no planejamento, organização e controle dos cuidados primários em saúde, fazendo uso dos recursos locais disponíveis. ${ }^{11}$

Neste contexto teórico, a proposição deste estudo foi avaliar a eficiência de um programa individualizado de exercícios para incontinência urinária executado no espaço domiciliar sob supervisão e avaliação sistemática do fisioterapeuta.

\section{MÉTODOS}

Realizou-se um ensaio clínico não randomizado, vinculado à pesquisa institucional "Estudo multidimensional de mulheres pós-menopausa do município de Catuípe/RS". Todas as mulheres que aceitaram participar da pesquisa assinaram o Termo de Consentimento Livre e Esclarecido e foram submetidas a avaliações físicas funcionais. O estudo foi aprovado pelo Comitê de Ética em Pesquisa da Universidade Regional do Noroeste do Estado do Rio Grande do Sul - UNIJUÍ, pelo parecer consubstanciado no 313/2011.

A população do estudo foi constituída por mulheres pós-menopausa, com idades entre 50 a 65 anos, residentes na área urbana do município de Catuípe, estado do Rio Grande do Sul. A amostra foi constituída por cinco mulheres. Para a seleção da amostra consideraram-se os seguintes critérios de inclusão: diagnóstico urofuncional de IU e adesão ao protocolo de intervenção constituído por TMAP e GH. Foram excluídas mulheres que não cumpriram integralmente o protocolo de intervenção.

$\mathrm{O}$ acesso à população ocorreu a partir da análise do banco de dados da pesquisa institucional para selecionar as mulheres que relatavam perda de urina. $\mathrm{Na}$ exploração do banco de dados identificaram-se 48 mulheres com queixa de perda de urina, das quais 21 foram contatadas, 11 compareceram ao encontro e apenas 6 realizaram a avaliação física funcional. Cinco mulheres finalizaram o protocolo. 
As variáveis de interesse do estudo foram: dados sociodemográficos, antecedentes obstétricos, caracterização da incontinência urinária representada por questões referentes a quando e como ocorria a perda de urina, dados sobre a micção (frequência, disúria, atraso, força abdominal, vontade, uso de protetores), mudanças de hábitos pela perda de urina, características sexológicas (dispareunia, vaginismo, anorgasmia e disorgasmia) e informações sobre a ingestão de líquidos (tipo e controle de ingestão).

O exame físico foi constituído de avaliação abdominal (inspeção de cicatrizes e de padrão respiratório; palpação para identificar algias, flatus, impactação fecal, tônus diafragmático e abdominal e diástase e teste da tosse) e avaliação perineal (inspeção do óstio da vagina, distância ânus-vulva, pregas anais e presença de matéria fecal, sensibilidade, motricidade, tônus e reflexos perineais e presença de prolapsos). Para avaliar a funcionalidade do assoalho pélvico foi aplicado o esquema PERFECT.

O esquema PERFECT é um protocolo validado por Laycock e Jerwood ${ }^{9}$ em que se avalia a funcionalidade da musculatura do assoalho pélvico através da mensuração da força/Power $(P)$, duração/endurance $(E)$, repetições/repetitions $(R)$ e número de repetições/ contrações rápidas/fast $(F)$.

A força/Power $(P)$ é avaliada pela contração voluntária máxima e graduada através da Escala de Oxford Modificada em uma escala de 0 a $5 .{ }^{12}$ Nos extremos, zero representa uma condição em que não é perceptível nenhuma contração muscular e 5 traduz uma contração forte em que há a compressão e elevação dos dedos contra forte resistência do examinador.

A duração/endurance (E) é avaliada solicitando-se à paciente contrair a musculatura perineal pelo maior tempo possível, sendo a mensuração dada pela contagem do tempo da sustentação da contração até que ocorra redução de $50 \%$ da contração voluntária máxima.

O número de repetições/repetitions $(R)$ é obtido através da solicitação de contrações voluntárias máximas, com 4 segundos de repouso entre elas; e o número de repetições/contrações rápidas/fast $(F)$ é obtido a partir da contração e relaxamento sem sustentação por 10 segundos. Com base nos resultados dessa avaliação, elaboram-se programas de exercícios individualizados.

As mulheres foram orientadas sobre todos os procedimentos da avaliação funcional, sendo que no momento da avaliação foi possível conscientizá-las quanto à percepção da musculatura perineal, o que foi importante para a execução do programa de exercícios. $\mathrm{O}$ programa individualizado foi composto por exercícios para o TMAP e GH e ocorreu no período de 12 semanas, com frequência de três vezes na semana.
Quinzenalmente cada participante fez uma consulta para orientação, supervisão e revisão/evolução dos exercícios. Para evitar a fadiga do períneo foi observada a alternância entre o TMAP e a GH. Para o TMAP avaliou-se a capacidade de contração das fibras musculares de contração rápida e lenta, o tempo de sustentação e a capacidade de repetição, sendo que todas realizaram três séries por exercício proposto. Já para a GH o exercício foi prescrito da mesma forma para todas as mulheres.

\section{RESULTADOS}

Participaram da pesquisa cinco mulheres, com média de idade de 60,2 $\pm 3,96$ anos. As características sociodemográficas encontram-se descritas na Tabela 1 e as características da incontinência urinária são apresentadas na Tabela 2.

Tabela 1. Características sociodemográficas de cinco pacientes submetidas a um programa individualizado de exercícios para incontinência urinária executado no espaço domiciliar.

\begin{tabular}{lc}
\hline \multicolumn{1}{c}{ Característica } & $\begin{array}{c}\text { Número de pacientes } \\
(\mathbf{n}=\mathbf{5})\end{array}$ \\
\hline Estado civil & 4 \\
$\quad$ Com companheiro & 1 \\
$\quad$ Sem companheiro & \\
Situação profissional & 2 \\
$\quad$ Aposentadas & 2 \\
Do lar & 1 \\
Em exercício & \\
Instrução & 3 \\
Ensino fundamental incompleto & 2 \\
Ensino fundamental completo & \\
Renda & 2 \\
Entre 1 e 2 salários mínimos & 1 \\
Mais de 2 salários mínimos & 2 \\
Não responderam &
\end{tabular}

Quando inquiridas se usavam ou não algum tipo de proteção, uma relatou usar absorvente diário em situações de gripe, quando se acentuam as crises de tosse e espirro. Questionou-se, também, o quanto a perda de urina interferia na vida das participantes, através de uma escala análoga visual que varia de zero a 10 , sendo zero quando a perda de urina não afeta a qualidade de vida e 10 quando a perda de urina afeta ao máximo a qualidade de vida. A pontuação referida pelas participantes variou de 4 a 9. Com relação a mudanças de hábito por conta da perda urinária, apenas uma relatou mudança. Quanto à ingestão líquida, duas pacientes relataram apenas controlar a ingestão de chimarrão. 
Tabela 2. Características da perda urinária de cinco pacientes submetidas a um programa individualizado de exercícios para incontinência urinária executado no espaço domiciliar.

\begin{tabular}{lc}
\hline Perda urinária & $\begin{array}{c}\text { Número de pacientes } \\
(\mathbf{n}=\mathbf{5})\end{array}$ \\
\hline Tipo de perda urinária & 3 \\
$\quad$ Mista & 2 \\
$\quad$ Somente a esforços & \\
Quantidade de perda & 4 \\
$\quad$ Molha a roupa & 1 \\
$\quad$ Não molha a roupa & \\
Caracterização da perda & 5 \\
$\quad$ Em jato & \\
Ocasião da perda & 2 \\
Precedida por vontade de urinar & 3 \\
Não precedida por vontade de urinar & 2 \\
$\quad$ Ocasional & \\
\hline
\end{tabular}

A Tabela 3 apresenta as características sexológicas e os antecedentes obstétricos, enquanto a avaliação da força, duração, repetição e número de repetições rápidas da musculatura do assoalho pélvico na pré e pós-intervenção está demonstrada na Tabela 4.

Para identificar se a perda de urina ocorre aos esforços ou em situações de instabilidade foi inquirido se os escapes ou a vontade de urinar aconteciam frente a esforços e em qual intensidade (grande, moderada ou pequena) ou quando havia situações de estresse, frio, barulho de torneira e mãos na água. Na pré-intervenção observou-se que $100 \%$ das mulheres relataram perda de urina ao tossir (grande esforço); uma apresentava escapes ao espirrar e uma ao caminhar rapidamente (moderados esforços). Nenhuma mulher relatou perda de urina ao andar ou trocar de posição (pequenos esforços). Na pós-intervenção persistiu a queixa de perda de urina em apenas uma das cinco mulheres que tinham escapes aos grandes esforços na préintervenção. A mesma taxa de resolução foi observada em relação aos esforços moderados.

Com relação à vontade e/ou perda em situações de instabilidade na avaliação pré-intervenção, a vontade urgente foi relatada nas condições de estresse por duas mulheres, no frio por duas e ao barulho de torneira por duas. A perda de urina ocorreu na condição de frio em duas, mãos na água em duas e barulho de torneira em uma. Na pós-intervenção a vontade urgente persistiu em situações de estresse em uma paciente e ao barulho de torneira aumentou para duas. Na pós-intervenção não foi verificada perda urinária em nenhuma dessas situações.
Tabela 3. Características sexológicas e obstétricas de cinco pacientes submetidas a um programa individualizado de exercícios para incontinência urinária executado no espaço domiciliar.

\begin{tabular}{lc}
\multicolumn{1}{c}{ Característica } & $\begin{array}{c}\text { Número de pacientes } \\
(\mathbf{n}=\mathbf{5})\end{array}$ \\
\hline Características sexológicas & 4 \\
Disorgasmia & 1 \\
Anorgasmia & 1 \\
Dispareunia e vaginismo & \\
Antecedentes obstétricos & 2 \\
2 filhos & 3 \\
4 filhos & 3 \\
Somente partos vaginais & 1 \\
Somente partos cesáreos & 1 \\
Partos vaginais e cesáreos & \\
\hline
\end{tabular}

Tabela 4. Comparação da avaliação da funcionalidade do assoalho pélvico através do esquema PERFECT, antes e após aplicação de um programa individualizado de exercícios para incontinência urinária executado no espaço domiciliar e sob supervisão de um fisioterapeuta.

\begin{tabular}{|c|c|c|}
\hline $\begin{array}{c}\text { Variáveis do esquema } \\
\text { PERFECT }\end{array}$ & $\begin{array}{l}\text { Pré-intervenção } \\
\text { (n) }(\text { n total=5) }\end{array}$ & $\begin{array}{l}\text { Pós-intervenção } \\
\text { (n) (n total=5) }\end{array}$ \\
\hline \multicolumn{3}{|l|}{ Power (Força)* } \\
\hline Contração fraca & 1 & - \\
\hline $\begin{array}{l}\text { Contração moderada } \\
\text { com alguma compressão } \\
\text { e elevação dos dedos }\end{array}$ & 4 & 2 \\
\hline $\begin{array}{l}\text { Contração boa com } \\
\text { compressão e elevação } \\
\text { dos dedos contra } \\
\text { resistência }\end{array}$ & - & 2 \\
\hline $\begin{array}{l}\text { Contração forte com } \\
\text { compressão e elevação } \\
\text { dos dedos contra forte } \\
\text { resistência }\end{array}$ & - & 1 \\
\hline \multicolumn{3}{|l|}{ Endurance (Sustentação) } \\
\hline 0 a 5 seg & 3 & 2 \\
\hline 6 a $10 \mathrm{seg}$ & 2 & 2 \\
\hline 11 a $15 \mathrm{seg}$ & - & 1 \\
\hline \multicolumn{3}{|l|}{ Repetitions (Repetições) } \\
\hline 0 a 10 repetições & 1 & 1 \\
\hline 11 a 20 repetições & 2 & 1 \\
\hline 21 a 30 repetições & 2 & 2 \\
\hline Acima de 30 repetições & - & 1 \\
\hline \multicolumn{3}{|l|}{ Fast (Rapidez) } \\
\hline 0 a 5 contrações rápidas & 3 & 2 \\
\hline 6 a 10 contrações rápidas & 2 & 3 \\
\hline
\end{tabular}

* Segundo Escala de OXFORD modificada. ${ }^{12}$ 
Comparando as avaliações pré e pós-intervenção evidenciou-se a redução de $80 \%$ de queixas de perda de urina ao esforço; redução de $40 \%$ na sensação de vontade de urinar em situações de estresse, frio, barulho de torneira e mãos na água; e presença de continência em $100 \%$ das mulheres em situação de instabilidade.

\section{DISCUSSÃO}

$\mathrm{Na}$ literatura encontram-se estudos que citam o espaço domiciliar como prolongamento do tratamento fisioterapêutico convencional, ou seja, o paciente realiza o tratamento na clínica com diferentes abordagens terapêuticas e é orientado a executar exercícios domiciliares, com o objetivo de maior eficácia do tratamento. ${ }^{13-15}$

Entretanto, estudos que preconizam o tratamento fisioterapêutico exclusivamente no domicílio são escassos. Sobretudo, não foi encontrada na literatura metodologia de intervenção semelhante à do presente estudo, o que pode ser a sua maior contribuição, divulgando uma experiência com possibilidade de reproduzi-la na atenção básica no espaço domiciliar.

A atenção domiciliar apresenta-se como uma ferramenta simples e de baixo custo, no entanto implica em uma postura profissional diferenciada em que o terapeuta empodera o paciente para que ele tenha condições de tomar decisões e corresponsabilizar-se pelo seu tratamento. Sem esta prerrogativa é certo que a intervenção domiciliar não alcançará o sucesso no tratamento planejado pelo terapeuta; trata-se aqui de uma relação de reciprocidade. Nessa perspectiva, o espaço domiciliar surge como uma estratégia de atenção à saúde diferenciada, podendo propiciar a aproximação dos profissionais de saúde com o paciente e seus familiares. ${ }^{10,16}$

O objetivo do presente estudo converge com essa concepção teórica e propõe um protocolo factível de ser executado por profissionais que atuam na atenção básica. A importância de estudos como este está no fato de que historicamente o profissional fisioterapeuta atuou na atenção secundária. Contudo, a política de saúde adotada nos últimos anos no país incluiu esse profissional nas equipes dos Núcleos de Apoio à Saúde da Família (NASF). Atuar nesse nível de atenção requer a produção de novas metodologias de trabalho que atendam de forma qualificada e resolutiva os problemas de saúde da população sob seus cuidados. Nesse contexto, o protocolo de pesquisa contribui com evidências de que métodos validados de tratamento podem ser usados na atenção básica a partir de novas metodologias.
$\mathrm{Na}$ busca por estudos desenvolvidos no espaço domiciliar e voltados ao tratamento da incontinência urinária destaca-se o de Dreher et al. ${ }^{17}$ Esse estudo foi realizado no domicílio da paciente e preconizou o uso de cones vaginais com supervisão e avaliação sistemática do fisioterapeuta. Os resultados da intervenção evidenciaram efetividade no tratamento da incontinência urinária, constatado pelo exame de urodinâmica.

Contrapondo aos achados de Dreher et al. ${ }^{17}$ encontra-se o estudo conduzido por Marques e Freitas. ${ }^{18}$ A pesquisa foi realizada com mulheres no período do climatério que constituíram dois grupos de intervenção. $O$ primeiro realizou sessões com o fisioterapeuta associado a informações através da cartilha para uso domiciliar; e o segundo grupo recebeu apenas orientações através da cartilha para o tratamento domiciliar. Os resultados mostraram que $75 \%$ das participantes submetidas ao tratamento domiciliar não cumpriram o protocolo devido ao esquecimento de executá-lo.

Já Baracho et al. ${ }^{13}$ associaram a terapia convencional para reeducação da continência urinária com exercícios realizados no domicílio com mulheres acima dos 60 anos. As mulheres eram submetidas a sessões de cinesioterapia e de eletroestimulação vaginal duas vezes na semana e eram orientadas a seguir exercícios diariamente no próprio domicílio. No que diz respeito à adesão das pacientes aos exercícios orientados no domicílio, os autores referem que não foi possível quantificar.

Ainda, Goode et al. ${ }^{19}$ propuseram um estudo comparativo de terapias para a IU e para tanto, constituíram três grupos. Desses, o grupo 1 e o grupo 2 foram submetidos a um protocolo que associou eletroestimulação com orientações domiciliares. O grupo 1 realizou biofeedback anorretal e o grupo 2 biofeedback anorretal e eletroestimulação transvaginal. A eletroestimulação teve como objetivo ensinar a paciente a realizar as contrações perineais de forma correta para posteriormente realizar no domicílio três vezes por dia. Para as atividades domiciliares eram realizadas orientações orais e escritas, uma vez por semana. O grupo 3 recebeu apenas orientações simples no primeiro encontro para realizar o programa de exercícios em casa. Os grupos 1 e 2 obtiveram resultados superiores ao grupo 3 , que foi submetido apenas à terapia comportamental sem orientação do profissional.

A divergência dos achados entre o presente estudo e o de Dreher et al. ${ }^{17}$ com os estudos de Marques, ${ }^{18}$ Baracho et al. ${ }^{13}$ e Goode et al. ${ }^{19}$ pode ser atribuída, em parte, à metodologia de supervisão e avaliação 
sistemática do fisioterapeuta, o que pressupõe que o terapeuta identifica de que forma o paciente está executando o exercício e faz as correções necessárias, bem como consegue progredir nos exercícios de forma a contemplar as especificidades de treinamento muscular, o que não é possível apenas pela orientação.

O acompanhamento e supervisão dos exercícios são fundamentais para a efetividade de tratamentos realizados no domicílio. Os resultados do estudo de Zanetti et al. ${ }^{20}$ corroboram com esta afirmação, pois a pesquisa avaliou o efeito dos exercícios para o fortalecimento do assoalho pélvico em mulheres com IU. As mulheres foram divididas em dois grupos, um recebeu supervisão do fisioterapeuta e outro não. O grupo que teve supervisão apresentou melhores resultados. ${ }^{20}$

Com relação ao método do TMAP utilizado na intervenção, encontrou-se na literatura a pesquisa realizada por Sherburn et al. ${ }^{21}$ Esse estudo trabalhou com grupos randomizados de mulheres acima de 65 anos, sendo um grupo submetido ao TMAP por 20 semanas e outro a treinamento vesical através de orientações. O TMAP demonstrou ser mais eficaz que o treinamento vesical no tratamento da IU em mulheres idosas. ${ }^{21}$ Esse resultado confirma a efetividade do TMAP, que aliada à supervisão e avaliação sistemática, permite a progressão do tratamento e legitima a metodologia proposta no presente estudo.

No entanto, este estudo tem como limitador o número de participantes. Os motivos para a baixa participação podem ser o preconceito e os tabus, o que se justifica, em parte, pela escolaridade, renda e cultura da população do estudo, já que em estudos observacionais com esta população a prevalência de IU é significativa, segundo dados de Berlezi et al. ${ }^{2}$ que realizaram estudo com a mesma população.

O protocolo aplicado promoveu o incremento da força muscular, tempo de sustentação da contração muscular, aumento da velocidade de contração e número de repetições da musculatura do assoalho pélvico. Este incremento repercutiu na melhora das condições de incontinência urinária. Fato que foi evidenciado pela redução de $80 \%$ de queixas de perda de urina ao esforço; redução de $40 \%$ na sensação de vontade de urinar em situações de estresse, frio, barulho de torneira e mãos na água; e na continência de $100 \%$ das mulheres em situação de instabilidade.

A maior contribuição deste estudo está na proposição de um protocolo de tratamento simples e de baixo custo, que se mostra efetivo e com possibilidade de atender um maior número de mulheres, seja para reabilitação ou prevenção, garantindo melhor qualidade de vida à mulher, especialmente a partir do climatério.

\section{REFERÊNCIAS}

1. Guarasi T. Incontinência urinária em mulheres climatéricas: estudo epidemiológico, clínico e urodinâmico [Tese]. Campinas, SP: Faculdade de Ciências Médicas da Universidade Estadual de Campinas; 2000.

2. Berlezi EM, Dal Bem A, Antonello C, Leite MT, Bertolo EM. Incontinência urinária em mulheres no período pósmenopausa: um problema de saúde pública. Rev Bras Geriatr Gerontol. 2009;12(2):159-73.

3. Sociedade Brasileira de Urologia. Segura Aí. I Campanha de Conscientização sobre Incontinência Urinária [Internet]. [capturado em 2013 jul 02]. Disponível em: http://www. incontinenciaurinaria.com.br/index.html.

4. Abrams P, Cardozo L, Fall M, Griffiths D, Rosier P, Ulmsten U, Van Kerrebroeck P, Victor A, Wein A; Standardisation Sub-Committee of the International Continence Society. The standardization of terminology in lower urinary tract function: Report from the standardization sub-committee of the International Continence Society. Urology. 2003 Jan;61(1):37-49.

5. Camillato ES, Barra AA, Silva Jr AL. Incontinência urinária de esforço: fisioterapia versus tratamento cirúrgico / Stress urinary incontinence: physiotherapy versus surgical treatment. Femina. 2012 Jul-Aug;40(4):187-94.

6. Dumoulin C, Hay Smith J. Pelvic floor muscle treatment versus no treatment, or inactive control treatments, for urinary incontinence in women. Cochrane Database Syst Rev. 2010 Jan 20;(1):CD005654.

7. Latorre GFS, Seleme MR, Resende APM, Berghmans B. Ginástica hipopressiva: evidências de uma alternativa ao treinamento da musculatura do assoalho pélvico de mulheres com déficit proprioceptivo local. Fisioter Bras. 2011 NovDec;12(6):463-6.

8. Price N, Dawood R, Jackson SR. Pelvic floor exercise for urinary incontinence: a systematic literature review. Maturitas. 2010;67:309-15.

9. Laycock J, Jerwood D. Pelvic floor muscle assessment: The PERFECT Scheme. Physiotherapy. 2001 Dec;87(12):631-42.

10. Duarte YAO, Diogo MJE. Atendimento domiciliar: um enfoque gerontológico. São Paulo: Atheneu; 2000.

11. Mazza MPR. A visita domiciliária como instrumento de assistência de saúde. Rev Bras Cresc Des Hum. 1994; $4(2): 60-8$.

12. Laycock J. Clinical evaluation of the pelvic floor. In: Schussler B, Laycock J, Norton P, Stanton SL, editors. Pelvic Floor Re-education. London: Springer-Verlag; 1994. p. 42-8.

13. Baracho ELLS, Dias RC, Saleme CS, Geo MS, Laranjeira CLS, Lima RSBC. Impacto sobre a quantidade de urina perdida de uma intervenção fisioterapêutica em idosas com incontinência urinária. Fisioter Pesq. 2006;13(1): 23-29.

14. Bernardes NO, Péres FR, Souza ELBL, Souza OL. Métodos de tratamento utilizados na incontinência urinária de esforço genuína: um estudo comparativo entre cinesioterapia e eletroestimulação endovaginal. Rev Bras Ginecol Obstet. 2000;22(1):49-54

15. Langoni CS, Valmorbida LA, Resende TLR. A introdução de atendimentos por fisioterapeutas em unidades da atenção primária em saúde. Rev Bras Prom Saúde. 2012;25(3): 261-70.

16. Paskulin LM, Dias VR. Como é ser cuidado em casa: as percepções os clientes. Rev Bras Enferm. 2002 MarApr;55(2):140-5. 
17. Dreher DZ, Berlezi EM, Strassburger SZ, El Ammar MZ. O fortalecimento do assoalho pélvico com cones vaginais: programa de atendimento domiciliar. Scientia Medica. 2009 Jan-Mar;19(1):43-9.

18. Marques KSF, Freitas PAC. A cinesioterapia como tratamento da incontinência urinária na Unidade Básica de Saúde. Fisioter Mov. 2005 Out-Dez;18(4):63-7.

19. Goode PS, Burgio KL, Locher JL, Roth DL, Umlauf MG, Richter HE, Varner RE, Lloyd LK. Effect of behavior training with or without pelvic floor electrical stimulation on stress incontinence in women: a randomized controlled trial. JAMA. 2003 Jul 16;290(3):345-52.

20. Zanetti MR, Castro R de A, Rotta AL, Santos PD, Sartori M, Girão MJ. Impact of supervised physiotherapeutic pelvic floor exercises for treating female stress urinary incontinence. Sao Paulo Med J. 2007 Sep 6;125(5):265-9.

21. Sherburn M, Bird M, Carey M, Bø K, Galea MP. Incontinence improves in older women after intensive pelvic floor muscle training: an assessor-blinded randomized controlled trial. Neurourol Urodyn. 2011 Mar;30(3):317-24. 